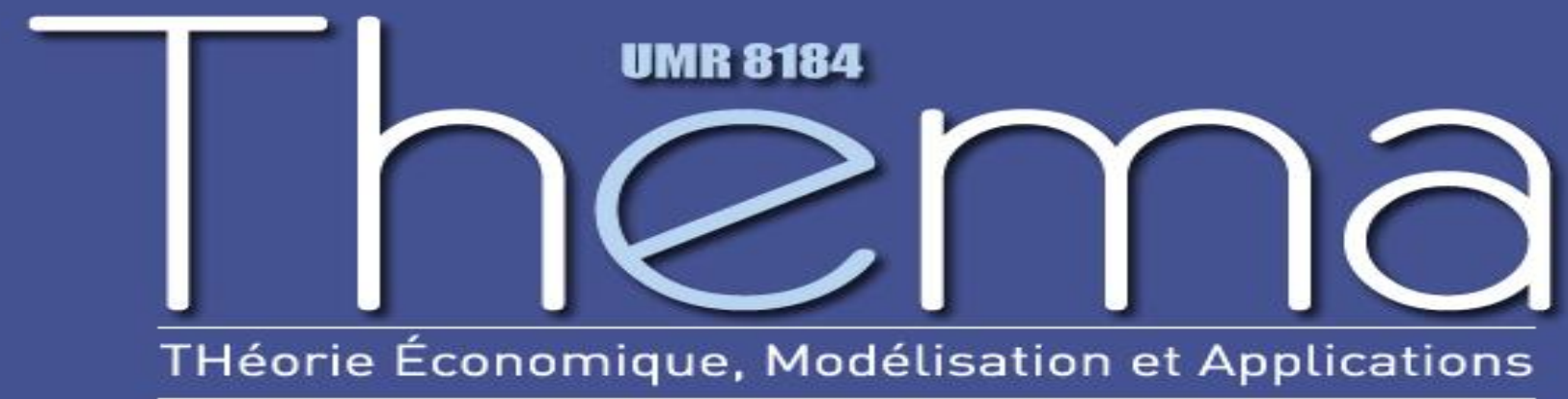

Thema Working Paper $\mathrm{n}^{\circ}$ 2013-04 Université de Cergy Pontoise, France

Politiques fiscales optimales pour les bas revenus et principe de compensation

Laurence Jacquet

Dirk Van de gaer

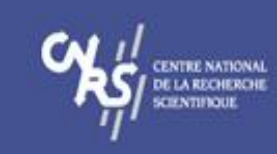

February, 2013 


\title{
Politiques fiscales optimales pour les bas revenus et principe de compensation*
}

\author{
Laurence Jacquet ${ }^{\dagger}$ \\ University of Cergy-Pontoise-THEMA
}

\author{
Dirk Van de gaer ${ }^{\ddagger}$ \\ Universiteit Gent et CORE
}

Février 2013

\begin{abstract}
Résumé
Nous considérons une économie composée d'agents faiblement ou hautement productifs qui diffèrent également dans leurs préférences pour le travail. Ces agents décident de participer ou non au marché du travail. Sous l'hypothèse d'asymétrie d'information, ce papier montre que le profil de taxation optimale dérivé avec le critère d'équivalence égalitarienne (Fleurbaey et Maniquet [2005, 2006]) satisfait toujours au principe de compensation (pour différents niveaux de productivité). Le critère de Roemer [1993, 1998] et le maximin pondéré à la Boadway et al. [2002] induisent, quant à eux, des barèmes fiscaux optimaux qui peuvent satisfaire au principe de compensation alors que les critères utilisés communément dans la littérature en taxation optimale y échouent. Des simulations numériques illustrent nos résultats analytiques et montrent comment les paramètres du modèle modifient la taxe sur la participation.
\end{abstract}

Mots clés: taxation optimale, principe de compensation, préférences hétérogènes pour le travail.

\section{Abstract}

We consider an economy in which agents differ in terms of productivity (that may be either high or low) as well as in their preferences for labour. Individuals decide whether or not they enter the labour force. In this context and under asymmetric information, the optimal tax schedules derived under the Egalitarian Equivalence criterion (Fleurbaey et Maniquet [2005, 2006]) always satisfy the compensation principle (for distinct productivity levels). Under the criterion of Roemer [1993, 1998] and the weighted maximin à la Boadway et al. [2002], the optimal tax schedules may satisfy the compensation principle while the standard criteria in the optimal taxation literature fail to do so. Numerical simulations illustrate our analytical results and highlight how the parameters in the model affect the participation tax.

Keywords: optimal taxation, compensation principle, heterogeneous preferences for labour.

JEL Classification: H21, D63

\footnotetext{
*Nous remercions tout particulièrement Marc Fleurbaey, Roland Iwan Luttens, Matohiro Sato et Alain Trannoy pour leurs commentaires.

${ }^{\dagger}$ Address: THEMA-University of Cergy Pontoise, 33 Boulevard du Port, Cergy-Pontoise, 95011 CEDEX, France. Laurence Jacquet est également membre du CESifo et de l'Université Catholique de Louvain (IRES et ETES). Courriel : laurence.jacquet@u-cergy.fr

${ }^{\ddagger}$ SHERPPA, Faculteit Economie en Bedrijfskunde, Universiteit Gent, Gent et Center of Operational Research and Econometrics (CORE), Louvain-la-Neuve, Belgique. Courriel : dirk.vandegaer@ugent.be.
} 


\section{Introduction}

Lorsque les individus diffèrent en termes de caractéristiques dont ils ne sont pas responsables et d'autres dont ils sont responsables, la littérature en choix social propose d'étudier l'égalité des opportunités à l'aune du critère de compensation. ${ }^{1}$ Celui-ci préconise que les inégalités résultant de facteurs qui ne relèvent pas de la responsabilité soient éliminées. Lorsque l'on suit l'hypothèse standard (en choix social appliqué) selon laquelle les individus sont responsables de leurs préférences mais pas de leur niveau de productivité, la compensation requiert que les individus avec des préférences identiques obtiennent un bien-être identique. ${ }^{2}$ Nous proposons ici un modèle dans lequel une condition nécessaire et suffisante pour que le principe de compensation soit satisfait consiste à saturer la contrainte d'incitation sur les travailleurs hautement qualifiés. Dès lors, lorsque le multiplicateur associé à cette contrainte est strictement positif, le principe de compensation est vérifié.

Ce papier montre que les critères communément utilisés en taxation optimale (une somme pondérée de transformations concaves des utilités individuelles dénommée critère welfariste, le maximin et le critère d'utilitarisme pondéré proposé par Boadway et al. [2002]) ne satisfont pas au principe de compensation, en asymétrie d'information. En revanche, les profils de taxation dérivés sous le critère développé par Roemer [1993, 1998] et le critère de maximin pondéré de Boadway et al. [2002] peuvent satisfaire ce principe. L'équivalence égalitarienne proposée par Fleurbaey et Maniquet [2005, 2006], quant à elle, permet toujours aux profils de taxation de satisfaire le principe de compensation. Nous illustrons ces résultats théoriques à l'aide de simulations numériques, en particulier, en mettant en évidence des configurations où le principe de compensation est satisfait ou non sous les critères de Roemer [1993, 1998] et de maximin pondéré.

Forts du résultat empirique que la marge extensive de l'offre de travail est très importante (Meghir et Phillips [2011]), nous supposons que les agents décident de participer ou non au marché du travail. Nous étudions comment les critères issus du choix social modifient la formule de taxation optimale de la participation que nous devons à Diamond [1980] et Saez [2002]. Nous dérivons la formule de taxation optimale de la participation sous les critères traditionnellement utilisés en taxation optimale et d'autres critères (l'utilitarisme pondéré à la Bodway et al. [2002], le maximin pondéré à la Boadway et al. [2002], le critère de Roemer et l'équivalence égalitarienne). Nous mettons en lumière qu'un terme nouveau, où apparaît le multiplicateur associé à la contrainte d'incitation sur les travailleurs hautement qualifiés, apparaît dans la formule générale et s'annule avec certains critères. En revanche, sous d'autres critères, ce terme est positif et le principe de compensation est alors satisfait. Nous dérivons une condition caractérisant l'optimum lorsque ce multiplicateur est positif.

Cet article s'appuie sur le cadre d'analyse de Jacquet et Van de gaer [2011] et prolonge ce

\footnotetext{
${ }^{1}$ Ce papier met l'accent sur le principe de compensation et les critères de choix social que nous étudierons en sont dérivés. Pour plusieurs auteurs (ex. Vandenbroucke [2001], Hild et Voorhoeve [2004]), le principe de compensation est la seule implication éthique de l'égalitarianisme sensible à la responsabilité. Néanmoins, un autre pan de littérature en philosophie, largement discuté en choix social, s'intéresse au principe de récompense qui nous dit comment redistribuer sur base des caractéristiques dont les individus sont responsables. Fleurbaey [2008] offre un excellent survol de cette littérature.

${ }^{2}$ La littérature en choix social suppose que les individus sont entièrement responsables soit de leurs préférences individuelles (qu'importe l'origine de ces dernières), soit des caractéristiques individuelles qu'ils contrôlent, cf. Fleurbaey [2008, p. 247 - 253]. Ces deux approches sont incompatibles. Nous suivons la première approche dans cet article.
} 
dernier. Il présente les justifications sous-jacentes à chaque critère normatif et les éventuelles questions éthiques que soulèvent certains critères. Jacquet et Van de gaer [2011] affirme que les profils de taxation obtenus avec de critères de choix social ne semblent pas contredire le principe de compensation. Cet article va plus loin en montrant que le critère d'équivalence égalitarienne satisfait toujours le principe de compensation tandis que les critères de Roemer et de maximin pondéré à la Boadway et al. [2002] peuvent ou non satisfaire ce principe. Le présent article dérive dès lors une condition caractérisant l'optimum lorsque le principe de compensation est satisfait, sous ces deux critères. En outre, nous montrons que, sous le critère de Roemer, lorsque l'économie est pauvre (c.-à-d. lorsque les dépenses gouvernementales exogènes sont élevées et les niveaux de productivité faibles), il est peu probable que le principe de compensation soit satisfait. Nos simulations mettent en lumière l'impact des différents paramètres du modèle sur la taxation optimale de la participation.

Cet article complète également les différents résultats de Fleurbaey et Maniquet en taxation optimale (résumés dans les chapitres 10 et 11 de Fleurbaey et Maniquet [2011]) qui reposent toujours sur l'hypothèse que l'offre de travail est le long de la marge intensive. En outre, notre analyse repose sur l'approche standard de maximisation d'un critère normatif sous contraintes budgétaire et d'incitation. Ceci nous permet de caractériser complètement le profil de taxation optimale contrairement à l'approche suivie par Fleurbaey et Maniquet. Ces derniers n'effectuent pas de comparaisons interpersonnelles de bien-être et construisent des fonctions d'ordre social à partir de propriétés normatives telles que le principe de Pareto et le principe de compensation. Dès lors, même s'il est possible de montrer que le profil de taxation optimale doit, par exemple, donner le plus grand subside aux travailleurs les moins bien rémunérés (cf. Fleurbaey et Maniquet [2006]), une caractérisation complète du profil de taxation optimale est impossible. Enfin, notre analyse compare les résultats obtenus sous des critères issus du choix social avec ceux obtenus sous des critères communément utilisés en taxation optimale.

La section 2 présente le modèle et le principe de compensation. La section 3 dérive la formule d'imposition optimale et détermine quel critère permet de satisfaire au principe de compensation. La section 4 illustre et complète nos résultats analytiques à l'aide de simulations numériques.

\section{Le modèle}

Nous considérons que la population a deux paramètres d'hétérogénéité, la productivité $w$ et la désutilité à travailler, $\alpha \in \mathbb{R}^{+}$. La productivité prend deux valeurs, $w_{H}>w_{L}>0$, qui correspondent aux salaires bruts dans les emplois hautement qualifiés et faiblement qualifiés, respectivement. Nous notons $F(\alpha)$ la fonction de répartition de $\alpha$, que nous supposons dérivable et strictement croissante. En outre, $\gamma$ (respectivement $1-\gamma$ ) représente la proportion d'individus faiblement (hautement) productifs dans la population. Nous supposons que la productivité et la désutilité du travail sont distribuées de façon indépendante. Dans le prolongement de Mirrlees [1971], nous considérons que les distributions de caractéristiques individuelles sont connues de tous, mais que la productivité et la désutilité d'un agent particulier constituent une information privée. 


\subsection{Comportement individuel}

Les agents décident de participer ou non au marché du travail. Leurs préférences sont représentées par la fonction d'utilité quasi-linéaire suivante $:^{3}$

$$
\begin{aligned}
& v(x)-\alpha \text { s'ils travaillent, } \\
& v(x) \text { s'ils ne travaillent pas, }
\end{aligned}
$$

où $x$ est la consommation, $v$ est une fonction de classe $C^{2}$ telle que $v(x): \mathbb{R}^{+} \rightarrow \mathbb{R}: x \rightarrow v(x)$ avec $v^{\prime}>0 \geq v^{\prime \prime}, \lim _{x \rightarrow \infty} v^{\prime}(x)=0, \lim _{x \rightarrow 0} v^{\prime}(x)=+\infty$ et $\lim _{x \rightarrow 0} v(x)=-\infty$. Chaque agent maximise son utilité compte tenu de sa contrainte budgétaire. La contrainte budgétaire d'un individu de productivité $w_{Y}(Y=L, H)$ s'écrit $x_{Y}=w_{Y}-T_{Y}(Y=L, H)$ où $T_{Y}$ est le niveau de taxation sur le revenu $w_{Y}$.

\subsection{Contraintes de participation et d'incitation}

Le profil de taxation dépend seulement du revenu brut $\left(0, w_{L}\right.$ or $\left.w_{H}\right)$. Dès lors, les instruments à la disposition du gouvernement sont trois niveaux de consommation $x_{u}, x_{L}$ et $x_{H}$, dénotant, respectivement, la consommation lorsqu'on est inactif, dans un emploi peu qualifié et dans un emploi hautement qualifié. Les agents peu productifs n'ont accès qu'aux emplois peu qualifiés tandis que les agents très productifs peuvent travailler dans des emplois peu ou hautement qualifiés. Dès lors, nous introduisons une contrainte pour inciter les agents hautement productifs à travailler dans des emplois hautement qualifiés. Cette contrainte d'incitation s'écrit :

$$
x_{H} \geq x_{L} .
$$

Comme toutes les fonctions objectifs que nous étudierons sont croissantes avec la consommation individuelle, violer cette contrainte induirait une perte de production qui aurait permis d'augmenter la consommation de chaque individu et donc la valeur de la fonction objectif.

Nous pouvons écrire les conditions de participation des agents comme suit. Un individu de productivité $w_{Y}$ et de désutilité à travailler $\alpha$ (avec $\left.Y=L, H\right)$ travaille ssi

$$
v\left(x_{Y}\right)-\alpha \geq v\left(x_{u}\right) \Leftrightarrow \alpha \leq \alpha_{Y}^{*}
$$

où $\alpha_{Y}^{*}$, le seuil au-delà duquel les agents de productivité $w_{Y}$ optent pour le non-emploi, est tel que $^{4}$

$$
v\left(x_{Y}\right)-\alpha_{Y}^{*}=v\left(x_{u}\right) \quad Y=L, H .
$$

De (1) et (2), nous pouvons écrire :

$$
\alpha_{H}^{*} \geq \alpha_{L}^{*} .
$$

\footnotetext{
${ }^{3}$ Si nous considérons des préférences plus générales du type $V(x, \alpha)$ avec $V$ est une fonction de classe $C^{2}$ telle que $V(x, \alpha): \mathbb{R}_{+}^{2} \rightarrow \mathbb{R}:(x, \alpha) \rightarrow v(x, \alpha)$ avec $V_{x}^{\prime}>0 \geq V_{x}^{\prime \prime}$ et $V_{\alpha}^{\prime \prime}<0, \lim _{x \rightarrow \infty} V_{x}^{\prime}(x, \alpha)=0, \lim _{x \rightarrow 0} V^{\prime}(x, \alpha)=+\infty$ et $\lim _{x \rightarrow 0} v(x, \alpha)=-\infty$, nos résultats ne sont pas qualitativement modifiés.

${ }^{4}$ L'ensemble des contraintes de participation et incitative pour chaque agent de type $\left(w_{Y}, \alpha\right)$ (avec $Y=L, H$ et $\alpha \in \mathbb{R}^{+}$) peut s'écrire comme (1)-(2). En outre, comme la décision d'offre de travail est binaire, le mécanisme (direct de révélation) qui implémente l'allocation optimale n'est pas complètement révélateur. Chaque agent révèle totalement sa productivité $w_{Y}$ mais pas sa valeur $\alpha$; il annonce uniquement si son $\alpha$ est supérieur ou non à $\alpha_{Y}^{*}$.
} 


\subsection{Principe de compensation}

Dans la lignée de la littérature appliquée en choix social (Fleurbaey [2008], Fleurbaey et Maniquet [2011]), nous supposons que les agents sont responsables de leur prédilection à travailler mais pas de leur productivité. ${ }^{5}$ Nous pouvons dès lors appliquer l'axiome de compensation, proposé dans Fleurbaey's [1994], à notre modèle. Ce principe exige que les différences de productivité ne se traduisent pas en divergence de bien-être individuel. En d'autres termes, ce principe requiert un bien-être identique pour des préférences identiques que nous abrégeons par BIPI.

Lemme 1 Une condition nécessaire et suffisante pour satisfaire BIPI est que $\alpha_{L}^{*}=\alpha_{H}^{*}$ qui est équivalent à $x_{L}=x_{H}$.

La preuve se trouve en annexe A. Pour satisfaire BIPI, les seuils $\alpha_{L}^{*}$ et $\alpha_{H}^{*}$ doivent être identiques ce qui revient à égaliser le consommation de tous les travailleurs, $x_{H}=x_{L}$. BIPI ne peut pas être satisfait, pour tout $\alpha$, dès que la contrainte (3) n'est pas saturée (c.-à-d. dès qu'il existe des $\alpha$ tels que $\alpha_{L}^{*}<\alpha<\alpha_{H}^{*}$ ).

\subsection{Préférences sociales et contrainte budgétaire}

Cette section présente différents critères normatifs étudiés soit dans la littérature en taxation optimale soit en choix social. La prochaine section dérivera les barèmes fiscaux optimaux obtenus sous ces différents critères.

\section{Welfarisme (utilitarisme, welfariste avec aversion pour l'inégalité et maximin)}

Dans la perspective welfariste, le bien-être social dépend uniquement des niveaux d'utilité individuels (Sen [1979]). L'objectif social welfariste aggrège les niveaux d'utilité individuels et peut souscrire à l'éthique égalitarienne en stipulant que les inégalités d'utilité entre individus sont socialement indésirables. Pour ce faire, l'objectif welfariste inclut une fonction concave croissante des niveaux d'utilité individuels. Nous étudions trois critères welfariste différents : l'utilitarisme $\left(S^{U}\right)$, l'objectif welfariste avec aversion pour l'inégalité $\left(S^{W}\right)$ et le maximin $\left(S^{M}\right)$. L'utilitarisme et le maximin sont deux formes extrêmes du welfarisme. L'utilitarisme suppose une aversion nulle pour l'inégalité tandis que le maximin suppose une aversion pour l'inégalité infinie. L'utilitarisme consiste à prendre la moyenne des niveaux d'utilité individuels. Le maximin consiste à maximiser le niveau d'utilité atteint par les plus mal lottis. ${ }^{6}$ En taxation optimale, ces trois objectifs sont très populaires. L'objectif utilitariste est supposé, pour exemples, dans Ebert [1992], Diamond et Sheshinski [1995], Boadway et al. [2000] et Hellwig [2007]. On trouve l'objectif welfariste avec aversion pour l'inégalité dans Mirrlees [1971], Atkinson et Stiglitz [1980], Diamond [1998], Choné et Laroque [2005], Kaplow [2008] et Kleven et al. [2009], pour ne citer que quelques exemples. Le critère maximin est quant à lui utilisé dans, par exemple, Atkinson [1975], Kanbur et Tuomala [1994], d'Autume [2001] et Boadway et Jacquet [2008].

\footnotetext{
${ }^{5}$ Nous adoptons ici l'hypothèse standard de la littérature appliquée qui s'intéresse à l'égalité des opportunités. Néanmoins, il serait possible de suivre la suggestion de Pestieau et Racionero [2009] et de décomposer le paramètre $\alpha$ en deux composants: $\alpha=\alpha_{P}+\alpha_{H}$. Les agents seraient responsables de leur $\alpha_{P}$ (un paramètre de préférence) mais non de leur $\alpha_{H}$ (un paramètre d'handicap). Si nous options pour cette approche, les résultats principaux de ce papier resteraient valides.

${ }^{6}$ Le critère maximin est communément appelé critère Rawlsien par les économistes, évidemment, cette inteprétation ne reflète pas de manière adéquate la richesse de la théorie de la justice de Rawls [1971].
} 
Dans notre contexte, le critère utilitariste s'écrit comme

$$
\begin{aligned}
& S^{U}=\gamma \int_{0}^{\alpha_{L}^{*}}\left[v\left(x_{L}\right)-\alpha\right] d F(\alpha)+\gamma \int_{\alpha_{L}^{*}}^{\infty} v\left(x_{u}\right) d F(\alpha) \\
& +(1-\gamma) \int_{0}^{\alpha_{H}^{*}}\left[v\left(x_{H}\right)-\alpha\right] d F(\alpha)+(1-\gamma) \int_{\alpha_{H}^{*}}^{\infty} v\left(x_{u}\right) d F(\alpha) .
\end{aligned}
$$

Appliqué à notre contexte, l'objectif welfariste s'écrit

$$
\begin{aligned}
& S^{W}=\gamma \int_{0}^{\alpha_{L}^{*}} \Psi\left(v\left(x_{L}\right)-\alpha\right) d F(\alpha)+\gamma \int_{\alpha_{L}^{*}}^{\infty} \Psi\left(v\left(x_{u}\right)\right) d F(\alpha) \\
& +(1-\gamma) \int_{0}^{\alpha_{H}^{*}} \Psi\left(v\left(x_{H}\right)-\alpha\right) d F(\alpha)+(1-\gamma) \int_{\alpha_{H}^{*}}^{\infty} \Psi\left(v\left(x_{u}\right)\right) d F(\alpha) .
\end{aligned}
$$

où la fonction $\Psi: \mathbb{R} \rightarrow \mathbb{R}: a \rightarrow \Psi(a)$ est strictement concave et traduit l'aversion à l'inégalité du gouvernement par rapport à la distribution des utilités dans la population.

Dans notre contexte, les contraintes d'incitation (2) mettent en évidence que ce sont les inactifs qui ont le bien-être le plus faible, $v\left(x_{u}\right)$. Dès lors, la fonction objectif maximin s'écrit

$$
S^{M}=v\left(x_{u}\right)
$$

La théorie de la taxation optimale a très largement supposé que les individus avaient les mêmes préférences et ne différaient que par leurs niveaux de productivité. Relâcher l'hypothèse de préférences identiques pose deux difficultés. La première, technique, réside dans l'étude des contraintes d'incitation lorsque les individus sont hétérogènes en plusieurs dimensions ce qui amène à traiter un problème de tamisage multidimensionnel. La seconde difficulté, normative, naît de l'argument selon lequel les inégalités de revenus ou de niveaux d'utilité qui naissent de goûts différents pour le travail ou de productivités différentes ne devraient pas être appréhendées de la même façon et faire l'objet du même élan redistributif (cf. Fleurbaey [2008] pour une présentation exhaustive de ce débat). Par exemple, avec le critère maximin, l'hétérogénéité des préférences peut susciter une controverse lorsqu'on détermine les agents les plus mal-lotis dont l'utilité sera maximisée. Le gouvernement peut hésiter à transférer des revenus vers des agents qui font le choix de ne pas travailler en raison de leurs préférences. En effet, ceci est contraire au principe de responsabilité. Le critère suivant a été conçu pour permettre au gouvernement d'attribuer des poids différents aux individus (par exemple, les plus mal-lotis) ayant des préférences différentes afin d'assurer plus ou moins de redistribution à leur égard.

\section{Boadway, Marchand, Pestieau et Racionero [2002]}

Boadway et al. [2002] propose une classe de critères normatifs qui, d'une part, redistribuent, parmi les individus ayant les mêmes préférences pour le loisir, des plus productifs vers les moins productifs et, d'autre part, attachent un poids aux utilités des individus selon leur goût pour le loisir. Intuitivement, il s'agit non seulement de compenser pour les différences de productivité au sein de chaque groupe d'individus ayant le même goût pour le loisir mais également de permettre au gouvernement de redistribuer davantage vers tel ou tel groupe de préférences en fonction du poids qu'il attribue à ce type de préférences. Ces critères adoptent donc des cardinalisations différentes des utilités individuelles selon les paramètres de préférences $\alpha$. 
Plus formellement, l'approche de Boadway et al. consiste à appliquer une transformation $G(v \mid \alpha)$ sur les utilités individuelles $v$, au sein de chaque groupe de préférences pour le loisir $\alpha$. En outre, des poids différents peuvent être attribués aux utilités d'agents ayant le même niveau de productivité mais des préférences distinctes pour le loisir. Les critères de Boadway et al. s'écrivent $\int_{a} W(\alpha) G(v \mid \alpha) d \alpha$ où $W(\alpha): \mathbb{R}^{+} \rightarrow \mathbb{R}^{+}: \alpha \rightarrow W(\alpha)$ est la fonction du poids social attribuée à un individu dont la désutilité au travail est $\alpha$. Boadway et al. [2002] supposent que la transformation $G(v \mid \alpha)$ est soit une somme (pondérée par les proportions d'agents), soit la fonction min. Dans le premier cas, le critère porte alors le nom de fonction de bien-être social utilitariste pondéré ("Weighted Utilitarianism"). Ce critère a été utilisé, pour exemple, dans Cremer et al. [2004, 2007]. Lorsque la transformation $G(v \mid \alpha)$ est la fonction min, le critère est alors appelé fonction de bien-être social maximin pondéré ("Weighted Maximin").

Dans notre modèle, la fonction de bien-être utilitariste pondéré ("Weighted Utilitarianism") de Boadway et al. [2002] s'écrit

$$
\begin{aligned}
& S^{W U}=\gamma \int_{0}^{\alpha_{L}^{*}} W(\alpha)\left[v\left(x_{L}\right)-\alpha\right] d F(\alpha)+\gamma \int_{\alpha_{L}^{*}}^{\infty} W(\alpha) v\left(x_{u}\right) d F(\alpha) \\
& +(1-\gamma) \int_{0}^{\alpha_{H}^{*}} W(\alpha)\left[v\left(x_{H}\right)-\alpha\right] d F(\alpha)+(1-\gamma) \int_{\alpha_{H}^{*}}^{\infty} W(\alpha) v\left(x_{u}\right) d F(\alpha) .
\end{aligned}
$$

La fonction de bien-être maximin pondéré ("Weighted Maximin") de Boadway et al. s'écrit quant à elle ${ }^{7}$

$$
S^{W M}=\int_{0}^{\alpha_{L}^{*}} W(\alpha)\left[v\left(x_{L}\right)-\alpha\right] d \alpha+v\left(x_{u}\right) \int_{\alpha_{L}^{*}}^{\infty} W(\alpha) d \alpha,
$$

comme détaillé dans l'annexe B.

\section{Roemer}

Le critère d'égalité des opportunités proposé par Roemer $[1993,1998]$ se concentre sur le principe de compensation BIPI. L'implémentation du principe de Roemer peut se concevoir comme une procédure en deux étapes. A la première étape, on classe les individus par leur niveau de responsabilité (leur préférence) et, si cela s'avère possible, on égalise les niveaux d'utilité à chaque niveau de responsabilité. Si une telle égalisation ne s'avère pas faisable, Roemer propose d'utiliser la fonction min. La seconde étape consiste à prendre la moyenne des minima obtenus à l'étape précédente. Fleurbaey [2008] rebaptise ainsi le critère de Roemer le critère de la moyenne des minima ("the mean of mins criterion"). Dans la littérature en taxation optimale, Bossert et al. [1999] et Schokkaert et al. [2004] dérivent les profils d'imposition linéaires optimaux sous le critère de Roemer.

Dans notre modèle, la contrainte (1) induit que pour tout $\alpha, v\left(x_{L}\right)-\alpha \leq v\left(x_{H}\right)-\alpha$. La fonction de Roemer s'écrit dès lors comme suit

$$
S^{R}=\int_{0}^{\alpha_{L}^{*}}\left[v\left(x_{L}\right)-\alpha\right] d F(\alpha)+\int_{\alpha_{L}^{*}}^{\infty} v\left(x_{u}\right) d F(\alpha) .
$$

Lorsque $W(\alpha)=f(\alpha)$ (pour tout $\alpha$ ), le critère de bien-être maximin pondéré de Boadway et al. (4) est le critère de Roemer.

\section{Equivalence égalitarienne}

\footnotetext{
${ }^{7}$ Soulignons que ce critère ne prend pas en compte les proportions de populations.
} 
Enfin, nous étudions le critère d'équivalence égalitarienne (EE) discuté dans Fleurbaey et Maniquet [2005, 2006]. Dans un modèle où l'offre de travail est modélisée le long de la marge intensive, Fleurbaey et Maniquet maximisent le salaire équivalent minimal. Ce critère naît de la combinaison de propriétés normatives telles que le principe de Pareto, une variante du principe de transfert de Pigou-Dalton ainsi qu'une version faible du principe de responsabilité appelée "sélection du laissezfaire". Selon ce principe normatif, si tous les individus avaient la même productivité, on opterait pour l'allocation de laissez-faire. L'idée sous-jacente est que les inégalités de revenus reflèteraient alors uniquement des différences de (libres) choix dues à l'hétérogénéité des préférences individuelles et que de tels choix doivent être respectés. La fonction de préférence sociale qui découle de ces principes consiste à indicer toute courbe d'indifférence en une allocation donnée par la pente du rayon qui part de l'origine (dans le plan où le travail est en abscisses et la consommation en ordonnées) et est tangente à cette courbe d'indifférence. Cette pente est appelée le salaire équivalent. Le salaire équivalent est ainsi défini comme le taux de salaire pour lequel un agent est indifférent entre son panier actuel et le panier qu'il peut atteindre avec ce salaire équivalent. Le calcul du salaire équivalent repose dès lors sur un choix contrefactuel d'offre de travail prenant une valeur dans l'intervalle $\left[0, \ell_{\max }\right]$ où $\ell_{\max }$ est l'offre de travail individuelle maximale (disons, en travaillant à temps plein).

Dans notre modèle en marge extensive, l'offre de travail individuelle peut uniquement prendre deux valeurs. Dès lors, le concept de salaire équivalent est ajusté en comparant le niveau de consommation actuel avec le salaire qui rendrait l'individu indifférent entre son panier actuel et le panier qu'il peut obtenir en travaillant. Pour les travailleurs, le salaire équivalent est simplement leur niveau actuel de consommation, $x_{Y}(Y=H$ or $L)$. Pour les inactifs, il s'élève à $v^{-1}\left(v\left(x_{u}\right)+\alpha\right)$, où $x_{u}$ est leur niveau de consommation actuel. Ainsi, le critère d'EE consiste à maximiser:

$$
S^{E E}=\min _{\alpha}\left\{x_{L}, x_{H}, v^{-1}\left(v\left(x_{u}\right)+\alpha\right)\right\} .
$$

Comme $v^{-1}$ (.) est une fonction croissante, le salaire équivalent est le plus faible pour les individus inactifs ayant le plus petit $\alpha$, c.-à-d. ceux dont le $\alpha=\alpha_{L}^{*}$. Dès lors, le plus faible salaire équivalent est $v^{-1}\left(v\left(x_{u}\right)+\alpha_{L}^{*}\right)=v^{-1}\left(v\left(x_{L}\right)\right)=x_{L}$. Ceci permet de réécrire le critère d'EE comme suit

$$
S^{E E}=x_{L}
$$

\section{Contrainte budgétaire}

La fonction d'imposition du gouvernement doit également satisfaire la contrainte budgétaire de l'Etat:

$$
\gamma\left[\left(w_{L}-x_{L}\right) F\left(\alpha_{L}^{*}\right)-x_{u}\left(1-F\left(\alpha_{L}^{*}\right)\right)\right]+(1-\gamma)\left[\left(w_{H}-x_{H}\right) F\left(\alpha_{H}^{*}\right)-x_{u}\left(1-F\left(\alpha_{H}^{*}\right)\right)\right]-R=0,
$$

où $R$ correspond au niveau de dépenses publiques exogènes ${ }^{8}$ qui peuvent être positives ou négatives. Cette contrainte budgétaire doit être saturée à l'optimum comme toutes les fonctions objectifs considérées dans ce papier sont croissantes en la consommation individuelle.

\footnotetext{
${ }^{8}$ Nous supposons $R<\gamma w_{L}+(1-\gamma) w_{H}$ de sorte que la contrainte budgétaire puisse être satisfaite.
} 


\section{Politique fiscale optimale et compensation}

Le barème optimal est celui qui maximise l'une des fonctions objectifs ci-dessus sous la contrainte budgétaire (6) et les contraintes (1) et (2). Nous définissons les élasticités de participation des agents peu productifs par rapport à $x_{L}$ comme suit

$$
\eta\left(x_{L}, \alpha_{L}^{*}\right) \equiv \frac{x_{L}}{F\left(\alpha_{L}^{*}\right)} f\left(\alpha_{L}^{*}\right) v^{\prime}\left(x_{L}\right) .
$$

Nous appelons $\lambda$ le multiplicateur de la contrainte budgétaire de l'État (6), $\mu_{L}$ et $\mu_{H}$ les multiplicateurs de la contrainte de participation des peu productifs et des hautement productifs respectivement (2) et $\nu$ le multiplicateur associé à la contrainte d'incitation (1). Ce multiplicateur est particulièrement intéressant car, lorsqu'il est positif $(\nu>0)$, nous savons, grâce au lemme 1 , que le principe de compensation BIPI est satisfait. En effet, $x_{H}$ et $x_{L}$ sont alors égaux.

En outre, l'utilité marginale sociale de la consommation des travailleurs faiblement productifs s'écrit :

$$
g_{L}^{X} \equiv \frac{S_{x_{L}}^{X}}{\lambda \gamma F\left(\alpha_{L}^{*}\right)},
$$

où $S^{X}$ représente l'un ou l'autre critère normatif où $X=U, W, M, W U, W M, R$ ou $E E$ (cf. Sous-section 2.4). Finalement, nous écrivons $T_{L}=w_{L}-x_{L}$ la taxe payée par les travailleurs peu productifs tandis que $T_{u}=-x_{u}$ est celle payée par les inactifs. Cette réécriture nous permet de définir $\left(T_{L}-T_{u}\right) / x_{L}$ comme le taux de taxation de la participation qui peut être positif $\left(T_{L}>T_{u}\right)$ ou négatif $\left(T_{u}>T_{L}\right)$. Dans ce dernier cas, les travailleurs reçoivent un transfert plus élevé que les inactifs.

Nous présentons à présent la formule d'imposition optimale sur les peu productifs. Soulignons que nous prenons la liberté de ne pas indicer les différentes variables de nos problèmes d'optimisation selon le critère normatif utilisé, à l'exception du poids social $g_{L}^{X}$.

Proposition 1 Le taux d'imposition optimale sur les peu productifs est tel que

$$
\frac{T_{L}-T_{u}}{x_{L}}=\frac{1}{\eta\left(x_{L}, \alpha_{L}^{*}\right)}\left[1-g_{L}^{X}+\frac{\nu}{\lambda \gamma F\left(\alpha_{L}^{*}\right)}\right],
$$

La formule de taxation optimale pour les hautement productifs est identique à (9) où les indices $H$ sont remplacés par $L$, où $1-\gamma$ se substitue à $\gamma$ et où on aura préalablement réalisé les mêmes substitutions dans l'élasticité (7) et l'utilité marginale sociale (8).

La proposition 1 étend la formule d'imposition optimale de Saez [2002] aux critères de choix social. Le taux d'imposition sur la participation à l'optimum est la combinaison d'un facteur lié à l'efficacité $1 / \eta\left(x_{L}, \alpha_{L}^{*}\right)$, d'un facteur éthique $1-g_{L}^{X}$ et d'un facteur lié aux incitants $\nu /\left(\lambda \gamma F\left(\alpha_{L}^{*}\right)\right)$. Ce dernier est nouveau et n'apparaît pas dans l'écriture de Saez [2002] (Equation (4), p.1048). Aussi, nous renvoyons à Saez [2002] pour le commentaire des facteurs d'efficacité et éthique et nous concentrons notre attention sur l'étude du facteur lié à la contrainte d'incitation.

La proposition suivante met en lumière que le multiplicateur $\nu$ associé à la contrainte d'incitation est nul sous les critères welfariste (avec aversion pour l'inégalité), maximin, utilitariste et utilitariste pondéré à la Boadway et al. La formule de taxation optimale (9) s'écrit alors comme chez Saez [2002]. Le multiplicateur $\nu$ est en revanche positif sous le critère d'équivalence égalitarienne et le dernier terme du membre de droite de (9) est alors positif. 
Proposition 2 Le multiplicateur associé à la contrainte d'incitation $\nu$ est nul avec les critères utilitariste, welfariste avec aversion pour l'inégalité, maximin et l'utilitarisme pondéré à la Boadway et al. En revanche, le multiplicateur $\nu$ est toujours positif avec le critère d'équivalence égalitarienne.

La preuve se trouve en annexe D. En outre, comme mis en évidence dans la même annexe, la contrainte d'incitation (1) n'est jamais saturée et ainsi, $x_{H}^{X}>x_{L}^{X}$ pour $X=U, W, M$ et $W M$. Dès lors, grâce au lemme 1, nous pouvons conclure sur la satisfaction ou non du principe de compensation sous les différents critères.

Corollaire 1 Le principe de compensation (BIPI) est toujours enfreint avec les critères welfariste, maximin, utilitariste et d'utilitarisme pondéré à la Boadway et al. En revanche, le principe de compensation (BIPI) est toujours satisfait avec le critère d'équivalence égalitarienne.

Ces résultats sont démontrés en annexe D. Intuitivement, supposons que $\nu>0$, de sorte que $x_{H}=x_{L}$, et prenons un $\varepsilon>0$ infinitésimal. Augmentons $x_{H}$ de $d x_{H}=\varepsilon /(1-\gamma)$ et réduisons $x_{L}$ d'un montant $d x_{L}=\varepsilon / \gamma$. Cette opération n'a aucun effet direct sur les critères normatifs $X=U, W, M$ et $W U$. En effet, lorsque $x_{H}=x_{L}$, les dérivées partielles de chacun de ces objectifs sociaux par rapport à $x_{H}$ (divisées par $1-\gamma$ ) et par rapport à $x_{L}$ (divisées par $\gamma$ ) sont identiques, $S_{x_{H}}^{X} /(1-\gamma)=S_{x_{L}}^{X} / \gamma$ (cf. annexe D). Cette opération, en revanche, augmente les recettes fiscales, qui peuvent être distribuées en respectant la contrainte d'incitation et les contraintes de participation de tous les agents, ce qui, de manière indirecte, augmente la valeur de ces fonctions objectifs. Dès lors, $x_{H}>x_{L}$ (et $\nu=0$ ) avec $X=U, W, M$ et $W U$. En revanche, sous le critère d'équivalence égalitarienne, l'augmentation de l'objectif social liée au supplément de recettes fiscales (redistribuées tout en respectant les contraintes de participation et la contrainte d'incitation) est plus que contrebalancé par l'effet négatif direct que la réduction de $x_{L}$ induit sur cet objectif social. En effet, la consommation $x_{H}$ n'apparait pas, contrairement à $x_{L}$, dans l'ojectif d'équivalence égalitarienne. Dès lors, à l'optimum, $x_{H}=x_{L}$. Le principe de compensation (BIPI) est donc toujours satisfait. En outre, l'annexe D montre que $\nu>0$ sous le critère d'équivalence égalitarienne.

Nous n'avons pas pu mettre en avant de résultats analytiques concernant $\nu$ et la satisfaction ou non du principe de compensation (c.-à-d. $x_{H}=x_{L}$ ), avec le critère de Roemer et le critère maximin pondéré à la Boadway et al. Ceci n'est pas surprenant car, comme le montreront nos simulations, avec les critères de Roemer et de maximin pondéré, $\nu$ peut être nul ou positif. La proposition suivante aide à fournir une intuition pour expliquer quand $\nu$ est nul ou positif.

Sous le critère d'équivalence égalitarienne, $\nu>0$ et le dernier terme de la formule de taxation (9) est non-nul. Avec les critères de Roemer et de maximin pondéré de Boadway et al., il est également possible que la contrainte d'incitation $x_{H} \geq x_{L}$ soit saturée et que $\nu>0$. Plus précisément, définissons $R_{H} \equiv(1-\gamma)\left[\left(w_{H}-x_{H}\right) F\left(\alpha_{H}^{*}\right)-x_{u}\left(1-F\left(\alpha_{H}^{*}\right)\right)\right]$, le revenu net collecté sur les individus hautement qualifiés. La proposition suivante décrit une condition que satisfait l'optimum social lorsque $\nu>0$.

Proposition 3 Sous les critères de Roemer, de maximin pondéré à la Boadway et al. et d'équivalence égalitarienne, nous avons $\nu>0$ si et seulement si $\partial R_{H} / \partial x_{H}<0$ (c-à-d si et seulement si augmenter $x_{H}$ réduit les dépenses gouvernementales) et $\nu=0$ si et seulement si $\partial R_{H} / \partial x_{H}=0$. 
La démonstration se trouve en annexe E. La proposition 3 nous apprend que, si, sous le critère de Roemer, de maximin pondéré à la Boadway et al. ou d'équivalence égalitarienne, ${ }^{9} \nu>0$ à l'optimum, la taxation sur les travailleurs hautement qualifiés ne maximise pas les recettes fiscales collectées sur ces travailleurs (c.-à-d. $\partial R_{H} / \partial x_{H}<0$ ). A contrario, $\nu=0$, sous le critère de Roemer et sous le critère maximin pondéré, si et seulement si la taxe sur les hautement qualifiés maximise les recettes fiscales sur ces derniers (c.-à-d. $\partial R / \partial x_{H}=0$ ).

La variable $x_{H}$ n'apparaît pas dans les critères de Roemer, de maximin pondéré et d'équivalence égalitarienne. Dès lors, lorsque le gouvernement réduit $x_{H}$, cela n'a pas d'impact direct sur la valeur de ces fonctions objectifs. Intuitivement, lorsque $\partial R_{H} / \partial x_{H}<0$, une augmentation de la taxation sur les hautement qualifiés augmente les recettes fiscales en provenance des individus hautement qualifiés, recettes qui servent à redistribuer davantage vers les travailleurs faiblement qualifiés ainsi que, pour le critère de Roemer (5) et le maximin pondéré (4), vers les inactifs. Cette redistribution se fait en respectant les contraintes d'incitation et de participation. Le gouvernement cherche à augmenter au maximum $R_{H}$, et donc à réduire au maximum $x_{H}$, mais la contrainte d'incitation (1) l'empêche de réduire $x_{H}$ en-deça de $x_{L}$. Ainsi, à l'optimum social, $\nu>0$ et $x_{H}=x_{L}$ vont de pair avec $\partial R_{H} / \partial x_{H}<0$. A contrario, $\nu=0$ et $x_{H}>x_{L}$ vont de pair avec $\partial R_{H} / \partial x_{H}=0$. Dans ce cas de figure, si le gouvernement ne réduit pas $x_{H}$ au niveau de $x_{L}$, c'est que, pour un $x_{H}$ supérieur à $x_{L}$, il a déjà maximisé le montant qu'il pouvait collecter des individus hautement qualifiés $\left(\partial R_{H} / \partial x_{H}=0\right)$ et ce faisant, il a déjà maximisé sa fonction objectif.

Dès lors, sous le critère de Roemer, que $\nu$ soit positif ou nul dépend de manière critique de la valeur marginale sociale des fonds publics $R_{H}$ qui est déterminée par les utilités marginales de la consommation des faiblement qualifiés, $v^{\prime}\left(x_{L}\right)$ et $v^{\prime}\left(x_{u}\right)$. Cette valeur sera d'autant plus élevée que les dépenses gouvernementales exogènes, $R$, sont importantes et que l'économie est peu productive (ce qui dépend de $w_{L}, w_{H}$ et $\gamma$ ). Lorsque $w_{L}, w_{H}$ sont faibles et que la population est composée de très peu d'individus hautement qualifiés (c.-à-d. que $\gamma$ est élevé), les niveaux de consommation des inactifs et des travailleurs faiblement qualifiés (que valorise le critère de Roemer) sont peu élevés. On s'attend alors à ce collecter le plus de taxes possible sur les hautement qualifiés soit souhaitable $\left(\partial R_{H} / \partial x_{H}=0\right)$ de sorte que le multiplicateur $\nu$ vaille zéro. Nos simulations (section 4) illustreront cette dernière propriété.

La proposition 2 a mis en évidence que le principe de compensation BIPI ne peut jamais être satisfait avec les préférences sociales communément utilisées en taxation optimale. En revanche, BIPI est toujours satisfait sous équivalence égalitarienne. Quant aux critères de Roemer et de maximin pondéré à la Boadway et al., ils n'excluent pas $x_{L}=x_{H}$ et peuvent donc satisfaire BIPI. La section suivante, au travers de simulations, met en exergue des cas où $x_{L}=x_{H}$ et d'autres où $x_{H}>x_{L}$ avec les critères de Roemer et de maximin pondéré.

\section{Simulations}

Les simulations de cette section illustrent et complètent nos résultats analytiques. Nous montrons comment les optima sont modifiés lorsque les paramètres principaux du modèle varient. En particulier, cette section donne des exemples où le principe BIPI est satisfait (et donc, $x_{L}=x_{H}$ ) sous

\footnotetext{
${ }^{9}$ Rappelons que, à l'optimum, $\nu$ est toujours positif avec le critère d'équivalence égalitarienne (cf. proposition 2).
} 
le critère de Roemer.

Nous supposons une fonction d'utilité logarithmique, $v(x)=\ln (x)$ et une distribution exponentielle des désutilités à travailler $\alpha \in \mathbb{R}^{+}$de moyenne et variance unitaires. Dès lors, $f(\alpha)=\exp (-\alpha)$ et $F(\alpha)=1-\exp (-\alpha)$. Il s'en suit que nous spécifions les différents critères normatifs comme suit.

Le critère utilitariste s'écrit

$$
S^{U}=\gamma\left[\exp \left(-\alpha_{L}^{*}\right)+\ln \left(x_{L}\right)-1\right]+(1-\gamma)\left[\exp \left(-\alpha_{H}^{*}\right)+\ln \left(x_{H}\right)-1\right] .
$$

Pour le critère welfariste, nous optons pour une aversion absolue à l'inégalité constante avec $\Psi(u)=-\exp (-\beta u) / \beta$ où $\beta>0$ est le paramètre d'aversion pour l'inégalité. Ceci nous permet de réécrire le critère welfariste comme suit :

$$
\begin{aligned}
& S^{W}=\frac{-\gamma}{\beta}\left[\frac{x_{L}^{-\beta}}{1-\beta}\left(1-\exp \left(\alpha_{L}^{*}(\beta-1)\right)\right)-\left(x_{u}\right)^{-\beta} \exp \left(-\alpha_{L}^{*}\right)\right] \\
& -\frac{(1-\gamma)}{\beta}\left[\frac{x_{H}^{-\beta}}{1-\beta}\left(1-\exp \left(\alpha_{H}^{*}(\beta-1)\right)\right)-\left(x_{u}\right)^{-\beta} \exp \left(-\alpha_{H}^{*}\right)\right] .
\end{aligned}
$$

Le critère maximin s'écrit simplement

$$
S^{M}=\ln \left(x_{u}\right) .
$$

Nous formulons le critère utilitariste pondéré à la Boadway et al., en premier, avec une fonction du poids social décroissante en choisissant $W(\alpha)=\exp (-\alpha)$ et, en second, avec une fonction du poids social croissante avec $W(\alpha)=1-\exp (-\alpha)$. Le critère utilitariste pondéré avec une fonction de poids décroissante s'écrit dès lors

$$
\begin{aligned}
& S^{W U d}=\gamma\left[\frac{1}{2} \ln \left(x_{L}\right)\left(1-\exp \left(-2 \alpha_{L}^{*}\right)\right)+\frac{1}{2} \alpha_{L}^{*} \exp \left(-2 \alpha_{L}^{*}\right)\right. \\
& \left.+\frac{1}{4}\left(\exp \left(-2 \alpha_{L}^{*}\right)-1\right)+\frac{1}{2} \ln \left(x_{u}\right) \exp \left(-2 \alpha_{L}^{*}\right)\right] \\
& +(1-\gamma)\left[\frac{1}{2} \ln \left(x_{H}\right)\left(1-\exp \left(-2 \alpha_{H}^{*}\right)\right)+\frac{1}{2} \alpha_{H}^{*} \exp \left(-2 \alpha_{H}^{*}\right)\right. \\
& \left.+\frac{1}{4}\left(\exp \left(-2 \alpha_{H}^{*}\right)-1\right)+\frac{1}{2} \ln \left(x_{u}\right) \exp \left(-2 \alpha_{H}^{*}\right)\right] .
\end{aligned}
$$

Le critère utilitariste pondéré avec une fonction de poids croissante s'écrit dès lors

$$
\begin{aligned}
& S^{W U c}=\gamma\left[\log \left(x_{L}\right)\left(\frac{1}{2}+\frac{1}{2} \exp \left(-2 \alpha_{L}^{*}\right)-\exp \left(-\alpha_{L}^{*}\right)\right)+\exp \left(-\alpha_{L}^{*}\right)\left(\alpha_{L}^{*}+1\right)\right] \\
& +\gamma\left[-\frac{1}{2} \exp \left(-2 \alpha_{L}^{*}\right)\left(\alpha_{L}^{*}+\frac{1}{2}\right)-\frac{3}{4}+\log \left(x_{u}\right)\left(\exp \left(-\alpha_{L}^{*}\right)-\frac{1}{2} \exp \left(-2 \alpha_{L}^{*}\right)\right)\right] \\
& +(1-\gamma)\left[\log \left(x_{H}\right)\left(\frac{1}{2}+\frac{1}{2} \exp \left(-2 \alpha_{H}^{*}\right)-\exp \left(-\alpha_{H}^{*}\right)\right)+\exp \left(-\alpha_{H}^{*}\right)\left(\alpha_{H}^{*}+1\right)\right] \\
& +(1-\gamma)\left[-\frac{1}{2} \exp \left(-2 \alpha_{H}^{*}\right)\left(\alpha_{H}^{*}+\frac{1}{2}\right)-\frac{3}{4}+\log \left(x_{u}\right)\left(\exp \left(-\alpha_{H}^{*}\right)-\frac{1}{2} \exp \left(-2 \alpha_{H}^{*}\right)\right)\right] .
\end{aligned}
$$

Le critère maximin pondéré à la Boadway et al. s'écrit :

$$
S^{W M}=\ln \left(x_{L}\right) \int_{0}^{\alpha_{L}^{*}} W(\alpha) d \alpha-\int_{0}^{\alpha_{L}^{*}} \alpha W(\alpha) d \alpha+\ln \left(x_{u}\right) \int_{\alpha_{L}^{*}}^{\infty} W(\alpha) d \alpha .
$$


Dans la section 2.4, nous avons vu que la fonction objectif maximin pondéré devient le critère de Roemer lorsque $W(\alpha)=f(\alpha)$. Lorsque les poids, décroissants, se définissent comme $W(\alpha)=$ $\exp (-\alpha)$, nous sommes précisément dans ce cas de figure ; $W(\alpha)=f(\alpha)$. Les résultats que nous obtiendrons sous le critère de Roemer seront donc également valides pour le critère maximin pondéré (avec poids décroissants).

Lorsque les poids sont croissants et s'écrivent $W(\alpha)=1-\exp (-\alpha)$, le critère maximin pondéré devient

$$
\begin{aligned}
& S^{W U c}=\ln \left(x_{L}\right)\left[\alpha_{L}^{*}-1+\exp \left(-\alpha_{L}^{*}\right)\right]-\left[\frac{\alpha_{L}^{* 2}}{2}-1+\exp \left(-\alpha_{L}^{*}\right)\left(\alpha_{L}^{*}+1\right)\right] \\
& +\ln \left(x_{u}\right)\left[|\alpha+\exp (-\alpha)|_{\alpha_{L}^{*}}^{\infty}\right] .
\end{aligned}
$$

dont le dernier terme tend vers l'infini. Notre fonction objectif n'est donc pas, en essence, bien définie. En effet, le support des $\alpha$ est $[0, \infty]$ de sorte que le poids attribué aux larges valeurs de $\alpha$ converge vers un nombre fini positif (dans notre cas, 1), et la valeur de la dernière intégrale dans $(10), \int_{\alpha_{L}^{*}}^{\infty}(1-\exp (-\alpha)) d \alpha$, explose. Nous n'étudierons dès lors pas plus en avant ce critère maximin pondéré dans nos simulations.

Le critère de Roemer devient

$$
S^{R}=\exp \left(-\alpha_{L}^{*}\right)+\ln \left(x_{L}\right)-1
$$

Enfin, le critère d'équivalence égalitarienne consiste simplement à maximiser

$$
S^{E E}=x_{L} .
$$

Nous présentons quatre ensembles de résultats, pour différentes valeurs de $w_{L}, w_{H}$ et $\gamma$. Le tableau 1 donne le cas de base avec $w_{L}=0.5, w_{H}=1.5$ et $\gamma=0.5$. Dans les autres tableaux, nous modifions certains des paramètres par rapport au cas de base.

Ainsi, dans le tableau 2, le différentiel de productivité entre les hautement et les faiblement qualifiés se réduit (en gardant la productivité moyenne, $\bar{w}$, constante à 1 ), $w_{H}=4 / 3$ et $w_{L}=2 / 3$. Dans le tableau 3, la fraction des faiblement qualifiés se réduit $(\gamma=1 / 3)$. Dans le tableau 4 , la fraction des faiblement qualifiés augmente $(\gamma=0.95)$.

Dans un premier temps, concentrons-nous sur les critères welfaristes qui correspondent aux cinq premières lignes dans chacun des tableaux. D'une ligne à l'autre, l'aversion pour l'inégalité augmente, d'une aversion nulle à la première ligne (avec l'utilitarisme), nous passons à une aversion infinie à la cinquième ligne (avec le maximin). Comme attendu de la proposition $2, \nu=0$ pour tous ces critères, dans tous les tableaux. En outre, la taxe sur la participation des faiblement qualifiés, $T_{L}-T_{u}$ est toujours positive. ${ }^{10}$

Les individus avec le plus faible niveau d'utilité sont toujours les personnes sans emploi (et les travailleurs pivots qui obtiennent la même utilité que les individus sans emploi). Sans surprise, dès lors, lorsque l'aversion pour l'inégalité augmente, la consommation des personnes sans emploi augmente, ce qui diminue la participation sur le marché du travail des deux types de travailleurs ( $\alpha_{L}^{*}$ et $\alpha_{H}^{*}$ se réduisent) et leurs niveaux de consommation.

\footnotetext{
${ }^{10} \mathrm{~L}$ 'article de Choné et Laroque [2005] a démontré analytiquement que le maximin exclut une taxation négative de la participation.
} 
Avec les critères welfaristes, lorsque nous comparons le tableau 1 avec le tableau 2, nous voyons que diminuer le différentiel de productivité réduit le niveau de consommation des inactifs, le différentiel des niveaux de consommation entre les hautement et les faiblement qualifiés ainsi que leurs taux de participation au marché du travail.

Aller du tableau 1 au tableau 3 (4) met en lumière que, lorsque la fraction de faiblement qualifiés diminue (augmente), la consommation de tous les agents augmente (diminue) et la participation au marché du travail se réduit (augmente).

Considérons, à présent, l'utilitarisme pondéré de Boadway et al. Les valeurs correspondant à l'utilitarisme (dont les poids sont identiques pour toutes les utilités) s'intercalent entre l'utilitarisme pondéré décroissant à la Bodway et al. (ligne 6) et l'utilitarisme pondéré croissant à la Bodway et al. (ligne 7). Comme attendu de la proposition 2, $\nu=0$ pour tous ces critères, dans tous nos tableaux. Lorsque les poids d'utilité sont décroissants en désutilité du travail, le planificateur social cherche à stimuler la participation sur le marché du travail. Ceci résulte en des subventions à la participation pour les travailleurs faiblement productifs $T_{L}-T_{u}<0$, dans tous nos tableaux.

En revanche, ce résultat ne prévaut pas avec l'utilitarisme standard ou l'utilitarisme à la Boadway et al. avec des poids croissants dans la désutilité du travail. Tandis que les poids deviennent plus croissants en la désutilité du travail (c.-à-d. lorsque l'on passe de l'utilitarisme pondéré décroissant à l'utilitarisme puis à l'utilitarisme pondéré croissant), la participation au marché du travail se réduit, la consommation des travailleurs diminue et la consommation des agents sans emploi augmente. Notre spécification avec des poids décroissants $(W(\alpha)=\exp (-\alpha))$ a des poids qui diminuent si rapidement que le planificateur social souhaite que quasiment tout le monde travaille. Pour ce faire, comme nous l'écrivions, il utilise des subventions à la participation. La participation au marché du travail est supérieure à $98 \%$ dans tous nos tableaux. Dès lors, les différences majeures entre les tableaux, sous l'utilitarisme pondéré décroissant, sont dues à des différences dans les productivités moyennes. Ceci explique pourquoi les différences de niveaux de consommation sont petites entre les tableaux 1 et 2 et larges entre les tableaux 1, 3 et 4 .

A présent, considérons le résultat sous le critère de Roemer (ligne 8) et d'équivalence égalitarienne (ligne 10).

Un premier résultat important concerne le multiplicateur $\nu$. Avec le critère de Roemer, le multiplicateur $\nu$ prend des valeurs positives dans les tableaux 1-3. Les hautement qualifiés sont taxés de façon à générer le maximum de recettes $\left(\partial R / \partial x_{H}=0\right)$ et grâce à la proposition 3 , nous savons que ceci donne $\nu>0$. En revanche, dans le tableau 4, où la fraction de faiblement qualifiés a drastiquement augmenté par rapport au tableau 1, nous trouvons $\nu=0$. Comme nous l'annoncions à la suite de la proposition 3, lorsque la productivité (moyenne) est faible et que la population est composée de très peu d'individus hautement qualifiés, le gouvernement souhaite collecter le plus de recettes fiscales possibles sur les personnes hautement qualifiées.

Sous le critère d'équivalence égalitarienne, comme attendu (cf. proposition 2), $\nu$ est toujours positif, dans tous nos tableaux. On peut montrer (cf. propositions F.1 et F.2 dans l'annexe F) qu'avec nos spécifications, pour le critère de Roemer ( $\operatorname{si} \nu>0$ ) et pour l'équivalence égalitarienne, les niveaux de consommation, à l'optimum, sont proportionnels à la productivité moyenne $\bar{w}$, de sorte que la participation au marché du travail est constante. Ceci explique pourquoi les niveaux des consommation et les participations au marché du travail (c.-à-d. $\alpha_{L}^{*}$ et $\alpha_{H}^{*}$ ) dans les tableaux 
1 et 2 (qui supposent la même productivité moyenne, $\bar{w}=1$ ) sont les mêmes, aussi bien avec le critère de Roemer que sous équivalence égalitarienne. Soulignons que, sous le critère d'équivalence égalitarienne, tous les individus entrent toujours sur le marché du travail. En outre, sous les critères de Roemer et d'équivalence égalitarienne, dans les tableaux 1 et 2 l'optimum social est tel que $x_{H}=x_{L}$ et la productivité moyenne, $\bar{w}$, vaut 1 . Dans le tableau 3 , la productivité moyenne passe à 1.167. Dès lors, comme précisé dans la proposition F.2 en annexe, la participation au marché du travail reste la même mais les niveaux de consommation dans le tableau 3 se multiplient par $\beta=1.167$ avec le critère de Roemer et l'équivalence égalitarienne.

Finalement, nous pouvons souligner que le critère d'équivalence égalitarienne conduit toujours à une subvention de la participation pour les faiblement qualifiés. Dans le contexte de Roemer, ceci se produit si la différence de productivité entre les travailleurs hautement et faiblement qualifiés est suffisamment large (cf. tableau 1 versus tableau 2) ou si la fraction de faiblement qualifiés n'est pas trop élevée (tableau 1 versus tableau 4).

\begin{tabular}{|llllllllll|}
\hline & $x_{u}$ & $x_{L}$ & $x_{H}$ & $\nu$ & $T_{L}-T_{u}$ & $\alpha_{L}^{*}$ & $\alpha_{H}^{*}$ & $F\left(\alpha_{L}^{*}\right)$ & $F\left(\alpha_{H}^{*}\right)$ \\
\hline Utilitarianisme & 0.300 & 0.726 & 1.009 & 0 & 0.074 & 0.884 & 1.213 & 0.587 & 0.703 \\
\hline Welfariste, $\beta=2$ & 0.339 & 0.649 & 0.895 & 0 & 0.190 & 0.650 & 0.972 & 0.478 & 0.622 \\
Welfariste, $\beta=4$ & 0.346 & 0.614 & 0.857 & 0 & 0.232 & 0.574 & 0.908 & 0.437 & 0.597 \\
Welfariste, $\beta=8$ & 0.350 & 0.557 & 0.822 & 0 & 0.293 & 0.465 & 0.855 & 0.372 & 0.575 \\
\hline Maximin & 0.350 & 0.545 & 0.805 & 0 & 0.305 & 0.444 & 0.833 & 0.358 & 0.565 \\
\hline Utilit. pond. décroissant & 0.016 & 0.991 & 1.008 & 0 & -0.475 & 4.099 & 4.116 & 0.983 & 0.984 \\
Utilit. pond. croissant & 0.343 & 0.590 & 0.915 & 0 & 0.253 & 0.543 & 0.982 & 0.419 & 0.625 \\
\hline Roemer & 0.277 & 0.871 & 0.871 & 0.258 & -0.095 & 1.147 & 1.147 & 0.682 & 0.682 \\
Maximin pond. décr. & & & & & & & & & \\
\hline Equiv. Egalitarienne & 0 & 1.000 & 1.000 & 0.5 & -0.5 & $\infty$ & $\infty$ & 1 & 1 \\
\hline
\end{tabular}

Tableau 1 : Cas de base: $w_{L}=0.5, w_{H}=1.5, \gamma=0.5$ et $\bar{w}=1$.

\begin{tabular}{|llllllllll|}
\hline & $x_{u}$ & $x_{L}$ & $x_{H}$ & $\nu$ & $T_{L}-T_{u}$ & $\alpha_{L}^{*}$ & $\alpha_{H}^{*}$ & $F\left(\alpha_{L}^{*}\right)$ & $F\left(\alpha_{H}^{*}\right)$ \\
\hline Utilitarianisme & 0.287 & 0.779 & 0.959 & 0 & 0.174 & 1.000 & 1.208 & 0.632 & 0.701 \\
\hline Welfariste, $\beta=2$ & 0.329 & 0.690 & 0.849 & 0 & 0.306 & 0.741 & 0.948 & 0.523 & 0.612 \\
Welfariste, $\beta=4$ & 0.336 & 0.652 & 0.810 & 0 & 0.351 & 0.663 & 0.879 & 0.485 & 0.585 \\
Welfariste, $\beta=8$ & 0.339 & 0.622 & 0.783 & 0 & 0.384 & 0.606 & 0.836 & 0.454 & 0.567 \\
\hline Maximin & 0.341 & 0.586 & 0.755 & 0 & 0.422 & 0.542 & 0.796 & 0.419 & 0.549 \\
\hline Utilit. pond. décroissant & 0.016 & 0.994 & 1.005 & 0 & -0.311 & 4.103 & 4.114 & 0.984 & 0.984 \\
Utilit. pond. croissant & 0.333 & 0.648 & 0.859 & 0 & 0.351 & 0.667 & 0.949 & 0.487 & 0.613 \\
\hline Roemer & 0.277 & 0.871 & 0.871 & 0.303 & 0.072 & 1.147 & 1.147 & 0.682 & 0.682 \\
Maximin pond. décr. & & & & & & & & & \\
\hline Equiv. Egalitarienne & 0 & 1.000 & 1.000 & 0.5 & -0.333 & $\infty$ & $\infty$ & 1 & 1 \\
\hline
\end{tabular}

Tableau 2: $w_{L}=2 / 3, w_{H}=4 / 3, \gamma=0.5$ et $\bar{w}=1$. 


\begin{tabular}{|llllllllll|}
\hline & $x_{u}$ & $x_{L}$ & $x_{H}$ & $\nu$ & $T_{L}-T_{u}$ & $\alpha_{L}^{*}$ & $\alpha_{H}^{*}$ & $F\left(\alpha_{L}^{*}\right)$ & $F\left(\alpha_{H}^{*}\right)$ \\
\hline Utilitarianisme & 0.343 & 0.811 & 1.113 & 0 & 0.032 & 0.862 & 1.178 & 0.578 & 0.692 \\
\hline Welfariste, $\beta=2$ & 0.389 & 0.725 & 0.985 & 0 & 0.164 & 0.621 & 0.927 & 0.463 & 0.604 \\
Welfariste, $\beta=4$ & 0.398 & 0.685 & 0.940 & 0 & 0.213 & 0.544 & 0.860 & 0.419 & 0.577 \\
Welfariste, $\beta=8$ & 0.402 & 0.650 & 0.909 & 0 & 0.252 & 0.482 & 0.817 & 0.382 & 0.558 \\
\hline Maximin & 0.403 & 0.603 & 0.876 & 0 & 0.300 & 0.404 & 0.776 & 0.332 & 0.540 \\
\hline Utilit. pond. décroissant & 0.019 & 1.155 & 1.172 & 0 & -0.636 & 4.089 & 4.103 & 0.983 & 0.984 \\
Utilit. pond. croissant & 0.394 & 0.647 & 0.994 & 0 & 0.247 & 0.496 & 0.925 & 0.391 & 0.604 \\
\hline Roemer & 0.323 & 1.016 & 1.016 & 0.360 & -0.193 & 1.147 & 1.147 & 0.682 & 0.682 \\
Maximin pond. décr. & & & & & & & & & \\
\hline Equiv. Egalitarienne & 0 & 1.167 & 1.167 & 0.666 & -0.667 & $\infty$ & $\infty$ & 1 & 1 \\
\hline
\end{tabular}

Tableau $3: w_{L}=0.5, w_{H}=1.5, \gamma=1 / 3$ et $\bar{w}=1.167$.

\begin{tabular}{|llllllllll|}
\hline & $x_{u}$ & $x_{L}$ & $x_{H}$ & $\nu$ & $T_{L}-T_{u}$ & $\alpha_{L}^{*}$ & $\alpha_{H}^{*}$ & $F\left(\alpha_{L}^{*}\right)$ & $F\left(\alpha_{H}^{*}\right)$ \\
\hline Utilitarisme & 0.157 & 0.468 & 0.673 & 0 & 0.189 & 1.094 & 1.456 & 0.665 & 0.767 \\
\hline Welfariste, $\beta=2$ & 0.181 & 0.414 & 0.603 & 0 & 0.267 & 0.830 & 1.206 & 0.564 & 0.701 \\
Welfariste, $\beta=4$ & 0.185 & 0.393 & 0.584 & 0 & 0.292 & 0.755 & 1.152 & 0.530 & 0.684 \\
Welfariste, $\beta=8$ & 0.186 & 0.376 & 0.573 & 0 & 0.310 & 0.703 & 1.123 & 0.505 & 0.675 \\
\hline Maximin & 0.187 & 0.358 & 0.562 & 0 & 0.329 & 0.651 & 1.100 & 0.478 & 0.667 \\
\hline Utilit. pond. décroissant & 0.009 & 0.549 & 0.564 & 0 & -0.040 & 4.089 & 4.112 & 0.983 & 0.984 \\
Utilit. pond. croissant & 0.178 & 0.230 & 0.416 & 0 & 0.388 & 0.489 & 0.836 & 0.385 & 0.566 \\
\hline Roemer & 0.153 & 0.476 & 0.504 & 0 & 0.176 & 1.136 & 1.189 & 0.679 & 0.696 \\
Maximin pond. décr. & & & & & & & & & \\
\hline Equiv. Egalitarienne & 0 & 0.55 & 0.55 & 0.050 & -0.048 & $\infty$ & $\infty$ & 1 & 1 \\
\hline
\end{tabular}

Tableau $4: w_{L}=0.5, w_{H}=1.5, \gamma=0.95$ et $\bar{w}=0.55$.

\section{Conclusion}

Cet article s'est intéressé au barème d'imposition optimale sur la participation lorsque le gouvernement n'observe pas la productivité et les préférences pour le travail de chaque contribuable. Nous avons montré que la formule générale de taxation optimale inclut un terme qui comprend le multiplicateur associé à la contrainte d'incitation des hautement qualifiés (qui évite que ces derniers ne travaillent dans des emplois peu qualifiés). Lorsque ce multiplicateur est positif, le principe de compensation ("bien-être identique pour préférences identiques") est satisfait. Lorsque la contrainte d'incitation n'est pas saturée, ce multiplicateur est nul et nous retrouvons la formule de taxation classique. Cela s'avère être le cas avec les critères welfaristes et avec l'utilitarisme pondéré de Boadway et al. [2002]. Nous avons montré que ce multiplicateur prend toujours une valeur positive sous le critère d'équivalence égalitarienne (Fleurbaey et Maniquet $[2005,2006]$ ) et qu'il peut prendre une valeur positive ou nulle avec le critère de Roemer et de maximin pondéré de Boadway et al. [2002]. Il n'y a donc que sous le critère d'équivalence égalitarienne que le principe de compensation est satisfait. Sous les critères de Roemer et de maximin pondéré de Boadway et al., il peut être satisfait, cela dépend de la richesse de l'économie (c.-à-d. de sa productivité moyenne et de ses ressources gouvernementales exogènes). Des simulations numériques ont illustré nos résultats analytiques. Elles montrent comment les paramètres du modèle modifient le multiplicateur associé à la contrainte d'incitation sous les critères de Roemer et de maximin pondéré et comment évolue la taxe sur la participation avec nos différents critères normatifs. 


\section{Références bibliographiques}

Atkinson A.B. [1975], 'Maxi-Min' et l'imposition optimale des revenus, Cahiers du Séminaire d'Econométrie, p. 74-86.

D’Autume A. [2001], L'imposition optimale du revenu : une application au cas français, Revue Française d' Économie, 15 (3), p. 3-63.

Atkinson A.B., Stiglitz J.E. [1980], Lectures on Public Economics, New York: Mc Graw-Hill. Boadway R. [2012], Review of Marc Fleurbaey and François Maniquet A Theory of Fairness and Social Welfare (New York: Cambride University Press 2011), Journal of Economic Litterature, Forthcoming.

Boadway R., Cuff K. et Marchand M. [2000], Optimal Income Taxation with Quasi-linear Preferences Revisited, Journal of Public Economic theory, 2(4), p. 435-460.

BoAdWAY R., JACQUET, L. [2008], Optimal marginal and average income taxation under maximin, Journal of Economic Theory, 143(1), p. 425-441.

Boadway R., Marchand M., Pestieau P. et Racionero M. [2002], Optimal redistribution with heterogeneous preferences for leisure, Journal of Public Economic Theory, 4(4), p. 475-498.

Bossert W., Fleurbaey M. et Van De Gaer D. [1999], Responsibility, talent, and compensation: a second best analysis, Review of Economic Design, 4(1), p. 35-55.

Chone, P. et Laroque G. [2005], Optimal Incentives for Labor Force Participation, Journal of Public Economics, 89(2-3), p. 395-425.

Cremer H., Lozachmeur J-M. et Pestieau P. [2004], Optimal retirement and disability benefits with audit, Finanzarchiv, 60(3), p. 278-295.

Cremer H., Lozachmeur J-M. et Pestieau P. [2007], Disability Testing and Retirement, The B.E. Journal of Theoretical Economics (Contributions), 7(1), Article 10.

Diamond P. [1980], Income Taxation with Fixed Hours of Work, Journal of Public Economics, 13(1), p. 101-110.

Diamond P. [1998], Optimal Income Taxation: An Example with a U-Shaped Pattern of Marginal tax Rates, American Economic Review, 88(1), p. 83-95.

Diamond P. et Sheshinski E. [1995], Economic aspects of optimal disability benefits, Journal of Public Economics, 57(1), p. 1-23.

Ebert U. [1992], A reexamination of the optimal nonlinear income tax, Journal of Public Economics, 49 , p. $47-73$.

Fleurbaey M. [1994], On fair compensation, Theory and Decision, 36, p. 277-307.

Fleurbaey M. [2008], Fairness, Responsibility and Welfare, Oxford: Oxford University Press.

Fleurbaey M., Maniquet F. [2005], Fair social orderings when agents have unequal production skills, Social Choice and Welfare, 24(1), p. 93-127.

Fleurbaey M., Maniquet, F. [2006], Fair Income Tax, Review of Economic Studies, 73(1), p. $55-83$.

Fleurbaey M., Maniquet, F. [2009], Compensation and responsibility, dans Arrow K.J., Sen A.K. et Suzumura K. (eds), Handbook of Social Choice and Welfare, vol. 2, Elsevier North Holland.

Fleurbaey M., Maniquet, F. [2011], A Theory of Fairness and Social Welfare, New York: Cambridge University Press, 293pp.

Hellwig M.F. [2007], A contribution to the theory of optimal utilitarian income taxation, Journal of Public Economics, 91(7-8), p. 1449-1477.

Hild M., Voorhoeve A. [2004], Equality of opportunity and opportunity dominance, Economics and Philosophy, 20, p. 117-146. 
JaCQuet L. et VAn DE Gaer, D. [2011], A comparison of optimal tax policies when compensation or responsibility matter, Journal of Public Economics, 95(11-12), p. 1248-1262.

Kanbur R. et Tuomala M. [1994], Inherent inequality and the optimal graduation of marginal tax rates, Scandinavian Journal of Economics, 96 (2), p. 275-282.

Kaplow L. [2008], Optimal Policy with Heterogeneous Preferences, The B.E. Journal of Economic Analysis 83 Policy, 8(1) (Advances), Art. 40.

Kleven H.J., Kreiner C.T. et Saez E. [2009], The Optimal Income Taxation of Couples, Econometrica, 77(2), p. 537-560.

Marchand M., Pestieau P. et del Mar Racionero M. [2003], Optimal Redistribution When Different Workers Are Indistinguishable, Canadian Journal of Economics, 36(4), p. 911-922.

Meghir C., Phillips, D. (2011), Labour Supply and Taxes, in The Mirrlees Review: Reforming the Tax System for the 21st Century, Oxford University Press (ed.), p. 202-274.

Mirrlees J. [1971], An Exploration in the Theory of Optimum Income Taxation, Review of Economic Studies, 38(2), p. 175-208.

Pestieau P. et Racionero M. [2009], Optimal redistribution with unobservable disability: welfarist versus non-welfarist social objectives, European Economic Review, 53(6), p. 636-644.

RAWLS J. [1971], A Theory of Justice, Oxford University Press, Oxford.

Roemer J. [1993], A pragmatic theory of responsibility for the egalitarian planner, Philosophy and Public Affairs, 22, p. 146-166.

Roemer J. [1998], Equality of Opportunity. Cambridge: Harvard University Press.

Saez E. [2002], Optimal Income Transfer Programs: Intensive versus Extensive Labor Supply responses, Quarterly Journal of Economics, 117(3), p. 1039-1073.

Schokkaert E., Van de gaer D., Vandenbroucke F. et Luttens R.I., [2004], Responsibility sensitive egalitarianism and optimal linear income taxation, Mathematical Social Sciences, 48(2), p. $151-182$.

Sen A.K. (1979), Utilitarianism and Welfarism, Journal of Philosophy, 76, p. 463-489.

VAN DE GAER D. [1993], Equality of opportunity and investment in human capital, PhD Thesis, KULeuven, Leuven, Belgium.

Vandenbroucke F. [2001], Social Justice and Individual Ethics in an Open Society. Equality, Responsability, and Incentives, Berlin: Springer-Verlag.

\section{Annexes}

\section{A Preuve du lemme 1}

Utilisons (3) et raisonnons par l'absurde en posant $\alpha_{H}^{*}>\alpha_{L}^{*}$. Il existe dès lors $\alpha, \alpha_{L}^{*}<\alpha<\alpha_{H}^{*}$ pour lequel des agents de type $w_{H}$ obtiennent $v\left(x_{H}\right)-\alpha$ comme utilité et des agents de type $w_{L}$ ont, quant à eux, $v\left(x_{u}\right)$. Comme l'utilité des agents de type $w_{H}$ dépend de $\alpha$ mais pas celle des agents de type $w_{L}$, il n'est pas possible d'égaliser les niveaux d'utilité pour tous les $\alpha, \alpha_{L}^{*}<\alpha<\alpha_{H}^{*}$. Le principe BIPI est dès lors enfreint. En outre, substituer $\alpha_{L}^{*}=\alpha_{H}^{*}$ dans (2) induit $x_{L}=x_{H}$ et vice-versa. 


\section{B La fonction de bien-être maximin pondéré de Boadway et al.}

La fonction de bien-être maximin pondéré de Boadway et al. consiste à sommer l'utilité des moinsbien lotis dans chaque groupe de préférences en lui ayant attribué un poids $W(\alpha)$. Pour les agents dont les préférences pour le loisir $\alpha \in\left[0, \alpha_{L}^{*}\right)$, les moins bien-lotis ont $v\left(x_{L}\right)-\alpha$ comme utilité. Pour les individus dont $\alpha \in\left[\alpha_{L}^{*}, \alpha_{H}^{*}\right]$, les moins bien-lotis obtiennent $v\left(x_{u}\right)$. Parmi les agents caractérisés par $\alpha \in\left[\alpha_{H}^{*},+\infty\right)$, les moins bien-lotis reçoivent également $v\left(x_{u}\right)$. Dès lors, le critère maximin pondéré s'écrit comme

$$
\begin{aligned}
& S^{W M}=\int_{0}^{\alpha_{L}^{*}} W(\alpha)\left[v\left(x_{L}\right)-\alpha\right] d \alpha+\int_{\alpha_{L}^{*}}^{\alpha_{H}^{*}} W(\alpha) v\left(x_{u}\right) d \alpha \\
& +\int_{\alpha_{H}^{*}}^{\infty} W(\alpha) v\left(x_{u}\right) d \alpha .
\end{aligned}
$$

qui peut se réécrire comme (4).

\section{Preuve de la proposition 1}

Le Lagrangien s'écrit

$$
\begin{aligned}
& £\left(x_{L}, x_{H}, x_{u}, \alpha_{L}^{*}, \alpha_{H}^{*}, \lambda, \mu_{L}, \mu_{H}, \nu, c\right)=S^{X}+\nu\left(x_{H}-x_{L}-c\right) \\
& +\lambda\left\{\gamma\left(w_{L}-x_{L}\right) F\left(\alpha_{L}^{*}\right)-\gamma x_{u}\left(1-F\left(\alpha_{L}^{*}\right)\right)\right. \\
& \left.+(1-\gamma)\left(w_{H}-x_{H}\right) F\left(\alpha_{H}^{*}\right)-(1-\gamma) x_{u}\left(1-F\left(\alpha_{H}^{*}\right)\right)-R\right\} \\
& +\mu_{L}\left[v\left(x_{L}\right)-\alpha_{L}^{*}-v\left(x_{u}\right)\right]+\mu_{H}\left[v\left(x_{H}\right)-\alpha_{H}^{*}-v\left(x_{u}\right)\right]
\end{aligned}
$$

Il est maximisé par rapport à $x_{L}, x_{H}, x_{u}, \alpha_{L}^{*}, \alpha_{H}^{*}$ et $c$, en prenant en considération que $c \geq 0$. Les conditions nécessaires s'écrivent

$$
\begin{aligned}
& S_{x_{L}}^{X}-\lambda \gamma F\left(\alpha_{L}^{*}\right)-\nu=-\mu_{L} v^{\prime}\left(x_{L}\right), \\
& S_{x_{u}}^{X}-\lambda\left[\gamma\left(1-F\left(\alpha_{L}^{*}\right)\right)-(1-\gamma)\left(1-F\left(\alpha_{H}^{*}\right)\right)\right]=\left(\mu_{L}+\mu_{H}\right) v^{\prime}\left(x_{u}\right), \\
& S_{x_{H}}^{X}-\lambda(1-\gamma) F\left(\alpha_{H}^{*}\right)+\nu=-\mu_{H} v^{\prime}\left(x_{H}\right), \\
& \lambda \gamma f\left(\alpha_{L}^{*}\right)\left(w_{L}-x_{L}+x_{u}\right)=\mu_{L}, \\
& \lambda(1-\gamma) f\left(\alpha_{H}^{*}\right)\left(w_{H}-x_{H}+x_{u}\right)=\mu_{H}, \\
& -\nu \leq 0 \text { et } \nu c=0 .
\end{aligned}
$$

Les équations (13), (16) et (7) permettent d'écrire (9).

\section{Preuve de la proposition 2 et du corollaire 1}

Nous démontrons, dans un premier temps, que $\nu=0$ pour $X=U, W, M, W U$. Notons que si $\nu>0,(18)$ induit $c=0$, de sorte que $x_{H}=x_{L}$. Substituant (17) dans (15), nous obtenons :

$$
\nu=-S_{x_{H}}^{X}+\lambda(1-\gamma)\left[F\left(\alpha_{H}^{*}\right)-f\left(\alpha_{H}^{*}\right)\left(w_{H}-x_{H}+x_{u}\right) v^{\prime}\left(x_{H}\right)\right] .
$$


De sorte que $\nu>0$ (et donc, $x_{H}=x_{L}=x$ et $\alpha_{H}^{*}=\alpha_{L}^{*}=\alpha^{*}$ ) implique que :

$$
F\left(\alpha^{*}\right)-\left(w_{H}-x+x_{u}\right) f\left(\alpha^{*}\right) v^{\prime}(x)>\frac{S_{x_{H}}^{X}}{\lambda(1-\gamma)} .
$$

De manière similaire, injecter (16) dans (13) donne :

$$
\nu=S_{x_{L}}^{X}-\lambda \gamma\left[F\left(\alpha_{L}^{*}\right)-f\left(\alpha_{L}^{*}\right)\left(w_{L}-x_{L}+x_{u}\right) v^{\prime}\left(x_{L}\right)\right],
$$

et nous trouvons que $\nu>0$ si et seulement si :

$$
F\left(\alpha^{*}\right)-\left(w_{L}-x+x_{u}\right) f\left(\alpha^{*}\right) v^{\prime}(x)<\frac{S_{x_{L}}^{X}}{\lambda \gamma}
$$

En supposant $x_{H}=x_{L}=x$ et $\alpha_{H}^{*}=\alpha_{L}^{*}$, nous calculons $S_{x_{H}}^{X}$ et $S_{x_{L}}^{X}$ pour $X=U, W, M$ et $W U$ :

$$
\begin{aligned}
& S_{x_{L}}^{U}=\gamma \int_{0}^{\alpha^{*}} v^{\prime}(x) d F(\alpha) \text { et } S_{x_{H}}^{U}=(1-\gamma) \int_{0}^{\alpha^{*}} v^{\prime}(x) d F(\alpha), \\
& S_{x_{L}}^{W}=\gamma v^{\prime}(x) \int_{0}^{\alpha^{*}} \Psi^{\prime}(v(x)-\alpha) d F(\alpha) \text { et } S_{x_{H}}^{W}=(1-\gamma) v^{\prime}(x) \int_{0}^{\alpha^{*}} \Psi^{\prime}(v(x)-\alpha) d F(\alpha), \\
& S_{x_{L}}^{M}=S_{x_{H}}^{M}=0 \\
& S_{x_{L}}^{W U}=\gamma v^{\prime}(x) \int_{0}^{\alpha^{*}} W(\alpha) d F(\alpha) \text { et } S_{x_{H}}^{B}=(1-\gamma) v^{\prime}(x) \int_{0}^{\alpha^{*}} W(\alpha) d F(\alpha) .
\end{aligned}
$$

Nous déduisons de ces équations que, si $\nu>0$, les membres de droite de (20) et (22) sont égaux; $S_{x_{H}}^{X} /(1-\gamma)=S_{x_{L}}^{X} / \gamma$. Dès lors, nous pouvons réécrire que $\nu>0$ exige :

$$
F\left(\alpha^{*}\right)-\left(w_{H}-x+x_{u}\right) f\left(\alpha^{*}\right) v^{\prime}(x)>F\left(\alpha^{*}\right)-\left[w_{L}-x+x_{u}\right] f\left(\alpha^{*}\right) v^{\prime}(x),
$$

mais ceci ne peut se vérifier que si $w_{H}<w_{L}$, ce qui est en contradiction avec l'hypothèse $w_{H}>w_{L}$. Nous pouvons dès lors conclure que pour $X=U, W, M$ et $W U$, on a $\nu=0$.

A présent, utilisons $\nu=0$ obtenu sous les critères $X=U, W, M$ et $W U$ et supposons que $x_{H}=x_{L}=x \Leftrightarrow \alpha_{H}^{*}=\alpha_{L}^{*}=\alpha^{*}$ (Lemma 1). Nous pouvons dès lors écrire (21) comme :

$$
\frac{S_{x_{L}}^{X}}{\gamma}=\lambda F\left(\alpha^{*}\right)-\frac{v^{\prime}(x)}{\gamma} \lambda \gamma f\left(\alpha^{*}\right)\left(w_{L}-x+x_{u}\right) .
$$

L'Equation (19) peut s'écrire comme :

$$
\frac{S_{x_{H}}^{X}}{1-\gamma}=\lambda F\left(\alpha^{*}\right)-\frac{v^{\prime}(x)}{1-\gamma} \lambda(1-\gamma) f\left(\alpha^{*}\right)\left(w_{H}-x+x_{u}\right)
$$

En outre, lorsque $x_{L}=x_{H}=x$, nous avons vu précédemment que $\widetilde{S}_{x_{L}}^{X} / \gamma=\widetilde{S}_{x_{H}}^{X} /(1-\gamma)$ pour $X=U, W, M$ et $W U$. Dès lors, les deux équations précédentes donnent $\lambda f\left(\alpha^{*}\right)\left(w_{L}-x+\right.$ $\left.x_{u}\right)=\lambda f\left(\alpha^{*}\right)\left(w_{H}-x+x_{u}\right)$. Cette dernière égalité ne peut prévaloir que si $w_{H}=w_{L}$, ce qui est une contradiction. Nous pouvons dès lors conclure que $x_{H}^{X}>x_{L}^{X}$ pour $X=U, W, M$ et $W U$. Combinant cette inégalité avec le lemme 1, il s'en suit que les critères $W, U, M$ et $W U$ enfreignent le principe de compensation BIPI.

Nous démontrons à présent que $\nu>0$ sous le critère d'équivalence égalitarienne $(E E)$. Partons de la situation où $x_{u}=0$. Nous avons alors $\alpha_{H}^{*}=\alpha_{L}^{*}=\infty$ de (2) et $\lim _{x \rightarrow 0} v(x)=-\infty$, ainsi que 
$x_{H}=x_{L}=\gamma w_{L}+(1-\gamma) w_{H}-R$ de (6). A présent, si nous augmentons $x_{u}$, de (2), nous déduisons que $\alpha_{H}^{*}$ et $\alpha_{L}^{*}$ doivent diminuer. Moins de revenus sont dès lors disponibles pour la redistribution parmi les travailleurs. Dès lors, $x_{L}$ ou $x_{H}$ diminue. Comme nous sommes partis de la situation où $x_{H}=x_{L}$ et comme la contrainte d'incitation s'écrit $x_{H} \geq x_{L}, x_{L}$ doit diminuer. Néanmoins, ceci ne peut être optimal avec un objectif social égalitarien équivalent car $x_{L}$ est justement maximisé. Dès lors, nous pouvons conclure qu'à l'optimum, $x_{u}=0$ et donc, $x_{H}=x_{L}=\gamma w_{L}+(1-\gamma) w_{H}-R$ doit être optimal avec un critère égalitarien équivalent.

A présent que nous avons démontré que $x_{u}$ valait zéro, nous pouvons prouver que $\nu>0$ à l'optimum. De la condition d'Inada, $\lim _{x \rightarrow 0} v^{\prime}(x)=\infty$, de $\alpha_{L}^{*}=\alpha_{H}^{*}=\infty$ et de (14), la condition du premier ordre par rapport à $x_{u}$, nous avons que $\mu_{L}$ et $\mu_{H}$ doivent valoir zéro. Mais alors, de (15), la condition du premier ordre par rapport à $x_{H}$, nous avons que $\nu=\lambda(1-\gamma)>0$.

\section{E Preuve de la proposition 3}

Avec les critères de maximin pondéré, de Roemer et d'équivalence égalitarienne, nous avons $S_{x_{H}}^{X}=$ 0 (avec $X=R, W M$ et $E E$ ). Substituons $S_{x_{H}}^{X}=0$ dans (15) qui, avec (17) donne :

$$
\nu=\lambda(1-\gamma)\left[F\left(\alpha_{H}^{*}\right)-f\left(\alpha_{H}^{*}\right)\left(w_{H}-x_{H}+x_{u}\right) v^{\prime}\left(x_{H}\right)\right]
$$

De la contrainte budgétaire (6), nous pouvons écrire :

$$
\frac{\partial R_{H}}{\partial x_{H}}=-(1-\gamma)\left[F\left(\alpha_{H}^{*}\right)-f\left(\alpha_{H}^{*}\right)\left(w_{H}-x_{H}+x_{u}\right) \frac{\partial \alpha_{H}^{*}}{\partial x_{H}}\right] .
$$

Comme $\alpha_{H}^{*}=v\left(x_{H}\right)-v\left(x_{u}\right)(\mathrm{cf} .(2))$, nous obtenons $\partial \alpha_{H}^{*} / \partial x_{H}=v^{\prime}\left(x_{H}\right)$ de sorte que $(23)$ devient

$$
\nu=-\lambda \frac{\partial R_{H}}{\partial x_{H}} .
$$

De cette dernière équation, nous déduisons que, pour les critères de maximin pondéré, de Roemer et d'équivalence égalitarienne, étant donné que $\lambda>0$, nous avons $\nu>0$ si et seulement si $\partial R_{H} / \partial x_{H}<$ 0 . En outre, nous obtenons $\nu=0$ uniquement si $\partial R_{H} / \partial x_{H}=0$. Comme $T_{H}=w_{H}-x_{H}$, cela revient à imposer une taxe sur les travailleurs qualifiés qui maximise les recettes fiscales que l'Etat obtient d'eux.

\section{F Preuves de la section 4}

Dans cette annexe, nous montrons qu'avec nos spécifications des simulations; $v(x)=\ln x, f(\alpha)=$ $\exp (-\alpha)$ et $R=0$, pour le critère de Roemer (si $\nu>0$ ) et pour l'équivalence égalitarienne, les niveaux de consommation, à l'optimum, sont proportionnels à la productivité moyenne $\bar{w}$, de sorte que la participation au marché du travail est constante. Dans un premier temps, nous montrons que l'optimum ne dépend que de la productivité moyenne (proposition F.1). Dans un second temps, nous montrons que la participation reste constante (proposition F.2).

Proposition F.1 Sous le critère équivalence égalitarienne et sous le critère de Roemer si l'optimum social est tel que $x_{H}=x_{L}$ (c-à-d. que BIPI est satisfait), l'optimum ne dépend que de la productivité moyenne $\bar{w}=\gamma w_{L}+(1-\gamma) w_{H}$. 
Preuve. A côté de la contrainte (1), les contraintes (2) et la contrainte budgétaire (6) peuvent, quant à elles, se réécrire comme suit :

$$
\begin{gathered}
\ln \left(x_{L}\right)-\alpha_{L}^{*}-\ln \left(x_{u}\right)=0, \\
\ln \left(x_{H}\right)-\alpha_{H}^{*}-\ln \left(x_{u}\right)=0, \\
\gamma\left[\left(w_{L}-x_{L}\right)\left[1-\exp \left(-\alpha_{L}^{*}\right)\right]-x_{u} \exp \left(-\alpha_{L}^{*}\right)\right], \\
+(1-\gamma)\left[\left(w_{H}-x_{H}\right)\left[1-\exp \left(-\alpha_{H}^{*}\right)\right]-x_{u} \exp \left(-\alpha_{H}^{*}\right)\right]=0 .
\end{gathered}
$$

De (24) et (25) nous obtenons :

$$
-\alpha_{L}^{*}=\ln \left(\frac{x_{u}}{x_{L}}\right) \text { and }-\alpha_{H}^{*}=\ln \left(\frac{x_{u}}{x_{H}}\right) .
$$

Insérant ces expressions dans la contrainte budgétaire gouvernementale (26), nous pouvons écrire

$$
\gamma\left[\left(w_{L}-x_{L}\right)\left[1-\frac{x_{u}}{x_{L}}\right]-x_{u} \frac{x_{u}}{x_{L}}\right]+(1-\gamma)\left[\left(w_{H}-x_{H}\right)\left[1-\frac{x_{u}}{x_{H}}\right]-x_{u} \frac{x_{u}}{x_{H}}\right]=0 .
$$

Supposons que $x_{H}=x_{L}=x\left(\Leftrightarrow \alpha_{H}^{*}=\alpha_{L}^{*}=\alpha^{*}\right.$, cf. lemme 1). La contrainte budgétaire (27) devient :

$$
\left(\gamma w_{L}+(1-\gamma) w_{H}\right)\left[1-\frac{x_{u}}{x}\right]-x\left[1-\frac{x_{u}}{x}\right]-x_{u} \frac{x_{u}}{x}=0 .
$$

Dans cette dernière équation, seule la productivité moyenne $\bar{w}=\gamma w_{L}+(1-\gamma) w_{H}$ joue un rôle sur les variables $x_{u}$ et $x$. En outre, dans les autres contraintes, (1), (24) et (25), aucun des paramètres $\left(w_{L}, w_{H}\right.$ et $\left.\gamma\right)$ ne joue un rôle. Etant donné que les critères de Roemer et d'équivalence égalitarienne, (11) et (12), ne dépendent pas non plus des paramètres $w_{L}, w_{H}$ et $\gamma$, l'optimum sous ces deux critères ne dépend que de la productivité moyenne.

Proposition F.2 Sous le critère d'équivalence égalitarienne et sous le critère de Roemer si l'optimum social est tel que $x_{H}=x_{L} \equiv x\left(\Leftrightarrow \alpha_{H}^{*}=\alpha_{L}^{*}=\alpha^{*}\right)$, multiplier la productivité moyenne par $\beta(>0)$ multiplie les valeurs optimale de $x_{u}$ et $x$ par $\beta$ et $\alpha^{*}$ ne se modifie pas.

Preuve. Le problème d'optimisation avec objectif d'équivalence égalitarienne, en y substituant $x_{H}=x_{L}=x\left(\Leftrightarrow \alpha_{H}^{*}=\alpha_{L}^{*}=\alpha^{*}\right)$, peut s'écrire :

$$
\operatorname{Max}_{\alpha^{*}, x_{u}, x} \exp \left(-\alpha^{*}\right)+\ln x-1,
$$

s. c. que :

$$
\begin{aligned}
\bar{w}\left(1-\frac{x_{u}}{x}\right) & -x\left(1-\frac{x_{u}}{x}\right)-\frac{\left(x^{u}\right)^{2}}{x}=0, \\
-\alpha^{*} & =\ln \left(\frac{x_{u}}{x}\right) .
\end{aligned}
$$

Nous pouvons substituer la dernière équation dans la fonction objectif et ré-écrire :

$$
\operatorname{Max}_{\alpha^{*}, x_{u}, x} \frac{x_{u}}{x}+\ln x-1,
$$

s. c. que :

$$
\bar{w}\left(1-\frac{x_{u}}{x}\right)-x\left(1-\frac{x_{u}}{x}\right)-\frac{\left(x_{u}\right)^{2}}{x}=0 .
$$


Le Lagrangien peut s'écrire comme suit :

$$
L(.) \equiv \frac{x_{u}}{x}+\ln x-1+\lambda\left\{\bar{w}\left(1-\frac{x_{u}}{x}\right)-x\left(1-\frac{x_{u}}{x}\right)-\frac{\left(x_{u}\right)^{2}}{x}\right\},
$$

où $\lambda$ est le multiplicateur associé à la contrainte budgétaire gouvernementale.

Les conditions du premier ordre par rapport à $x_{u}, x$ et $\lambda$ sont :

$$
\begin{aligned}
\frac{1}{x}+\lambda\left[-\frac{\bar{w}}{x}+1-\frac{2 x_{u}}{x}\right] & =0, \\
-x_{u} x^{-2}+\frac{1}{x}+\lambda\left[\frac{\bar{w} x_{u}}{x^{2}}-1+\frac{\left(x_{u}\right)^{2}}{x^{2}}\right] & =0, \\
\bar{w}\left(1-\frac{x_{u}}{x}\right)-x\left(1-\frac{x_{u}}{x}\right)-\frac{\left(x_{u}\right)^{2}}{x} & =0 .
\end{aligned}
$$

Nous multiplions les deux membres de la première équation par $x(\neq 0)$ et nous multiplions les deux membres de la seconde équation par $x^{2}(\neq 0)$. Ensuite, nous divisons les deux membres de la nouvelle première équation par les deux membres de la seconde équation que nous avons obtenue et nous pouvons écrire :

$$
\begin{aligned}
& \frac{1}{-x_{u}+x}=\frac{-\bar{w}+x-2 x_{u}}{\bar{w} x_{u}-x^{2}+\left(x_{u}\right)^{2}} \\
& \Leftrightarrow\left(x_{u}\right)^{2}-\bar{w} x-3 x_{u} x+2 x^{2}=0 .
\end{aligned}
$$

Nous avons ainsi un système de deux équations à deux inconnues, $x$ and $x_{u}$ :

$$
\begin{aligned}
& \left(x_{u}\right)^{2}-3 x_{u} x+2 x^{2}=\bar{w} x, \\
& x^{2}-x_{u} x+\left(x_{u}\right)^{2}=\bar{w}\left(x-x_{u}\right) .
\end{aligned}
$$

Nous écrivons les solutions de ces deux équations comme $\widehat{x_{u}}$ and $\widehat{x}$ :

$$
\begin{aligned}
& \left(\widehat{x_{u}}\right)^{2}-3 \widehat{x_{u}} \widehat{x}+2 \widehat{x}^{2}=\bar{w} \widehat{x}, \\
& \widehat{x}^{2}-\widehat{x_{u}} \widehat{x}+\left(\widehat{x_{u}}\right)^{2}=\bar{w}\left(\widehat{x}-\widehat{x_{u}}\right) .
\end{aligned}
$$

A présent, multiplions la productivité moyenne $\bar{w}$ par $\beta$. Remplacer $\bar{w}$ par $\beta \bar{w}$ dans les conditions du premier ordre (30) et (31) donne :

$$
\begin{aligned}
& \left(x_{u}\right)^{2}-3 x_{u} x+2 x^{2}=\beta \bar{w} x, \\
& x^{2}-x_{u} x+\left(x_{u}\right)^{2}=\beta \bar{w}\left(x-x_{u}\right) .
\end{aligned}
$$

Prenons, à présent, les valeurs de $\widehat{x_{u}}$ et $\widehat{x}$ multipliées par $\beta$ et substituons-les dans les conditions du premier ordre ci-dessus :

$$
\begin{aligned}
& \beta^{2}\left[\left(\widehat{x_{u}}\right)^{2}-3 \widehat{x_{u}} \widehat{x}+2 \widehat{x}^{2}\right]=\beta^{2} \bar{w} \widehat{x}, \\
& \beta^{2}\left[\widehat{x}^{2}-\widehat{x_{u}} \widehat{x}+\left(\widehat{x_{u}}\right)^{2}\right]=\beta^{2} \bar{w}\left(\widehat{x}-\widehat{x_{u}}\right) .
\end{aligned}
$$

En vertu de la définition de $\widehat{x_{u}}$ et $\widehat{x}$ dans (32) et (33), ces nouvelles valeurs satisfont les conditions du premier ordre et sont donc optimales. Substituer ces nouvelles valeurs dans (29) (où $\widehat{x_{u}}$ et $\widehat{x}$ se substituent à $x_{u}$ et $x$ ) permet de démontrer que $\alpha^{*}$ ne se modifie pas.

On démontrera de même que, sous le critère de Roemer, si l'optimum social est tel que $x_{H}=$ $x_{L} \equiv x\left(\Leftrightarrow \alpha_{H}^{*}=\alpha_{L}^{*}=\alpha^{*}\right)$, multiplier la productivité moyenne par $\beta(>0)$ multiplie les valeurs optimale de $x_{u}$ et $x$ par $\beta$ et, qu'en outre, $\alpha^{*}$ ne se modifie pas. 